\title{
Epidemiological and clinical characteristics analysis of 11 children with 2019 novel coronavirus infection in Chongqing: a single-center retrospective study
}

\author{
Qiang Chen", Xiuying Tian", Yarui Luo, Jun Liu, Li Jiang, Xiaoqian Feng, Zongwen Chen \\ Department of Pediatrics, Chongqing University Three Gorges Hospital, Chongqing, China \\ Contributions: (I) Conception and design: X Feng, Z Chen; (II) Administrative support: L Jiang; (III) Provision of study materials or patients: J Liu; (IV) \\ Collection and assembly of data: X Tian, Y Luo, Q Chen; (V) Data analysis and interpretation: Q Chen; (VI) Manuscript writing: All authors; (VII) \\ Final approval of manuscript: All authors. \\ \#These authors contributed equally to this work. \\ Correspondence to: Xiaoqian Feng; Zongwen Chen. Chongqing University Three Gorges Hospital, 165 Xincheng Road, Wanzhou District, \\ Chongqing 404000, China. Email: 158681536@qq.com; 54964672@qq.com.
}

Background: The global epidemic of the 2019 novel coronavirus (2019-nCoV) is still going on. This article shares information about the infected children from a treatment center in Chongqing, China.

Methods: A retrospective analysis of the epidemiology, clinical symptoms, signs, laboratory examinations, chest computed tomography results, treatment effect of 11 children infected by 2019-nCoV was performed. Children were diagnosed from January 25 to February 29, 2020 in Chongqing University Three Gorges Hospital.

Results: The mean age of the 11 children with 2019-nCoV infection was 11 years and 5 months. Two cases $(18 \%)$ were imported cases from Wuhan. The 9 cases $(82 \%)$ were family cluster cases. There were 5 asymptomatic type cases (45\%), 2 mild cases (18\%), and 4 common type cases (37\%). The most common symptom was fever ( 5 cases), cough (3 cases), sore throat (1 case) and diarrhea (1 case). There were abnormal chest CT changes in 6 cases, including 4 cases with patchy ground-glass opacities and 2 cases with thickened lung texture. Laboratory tests showed that procalcitonin increased in 4 cases (36\%), and C-reactive protein (CRP) increased in 1 case (9\%). In lymphocyte subgroup examination, lymphocyte count increased in 2 cases $(18 \%)$ and decreased in 1 case $(9 \%)$; T\%, cluster of differentiation 8 (CD8)+ T\%, and natural killer (NK) cell\% were normal in 11 cases; CD4+ T\% was increased in 2 cases (18\%), and CD4+ T\%/CD8+ T\% was decreased in 1 case (9\%); B\% was increased in 1 case $(9 \%)$. The interleukin 4 (IL-4), IL-10, and IL17 in 11 cases were normal; IL-6 was increased in 7 cases (64\%); tumor necrosis factor-alpha (TNF- $\alpha$ ) was increased in 1 case (9\%); and interferon gamma (IFN- $\gamma$ ) was increased in 6 cases $(55 \%)$. All patients had been discharged from the hospital.

Conclusions: Children are generally susceptible to $2019-\mathrm{nCoV}$, and the main way of infection is close contact with an infected person in the family. Clinical symptoms are mild. Laboratory and chest CT examinations are not as typical as those of adults. The prognosis is generally good. The unique immune function of children may help fight the new coronavirus.

Keywords: 2019-nCoV; children; immunity

Submitted Nov 12, 2020. Accepted for publication Dec 17, 2020.

doi: $10.21037 /$ tp-20-429

View this article at: http://dx.doi.org/10.21037/tp-20-429 


\section{Introduction}

At the end of 2019, a new virus was reported and then classified as a novel coronavirus by the World Health Organization [the 2019 novel coronavirus, (COVID-19)] (1). On January 30, 2020, the World Health Organization (WHO) listed novel coronavirus pneumonia as a "public health emergency of international concern". The global epidemic of the 2019 novel coronavirus (2019$\mathrm{nCoV}$ ) is still going on. Although the initial diagnosis and treatment guidelines indicated that middle-aged and elderly people were susceptible and that children were less susceptible, as the epidemic has developed, there have been reports of pediatric cases. The number of pediatric patients has gradually increased, and the infection has affected premature infants and infants, with some falling critically ill. Therefore, in the later version of diagnosis and treatment guidelines, those susceptible to COVID-19 have been expanded to include the entire population (2). Based on official statistics, relative to the current total number of affected people, the total number of pediatric patients accounts for a low proportion. Due to the unique growth, development, and immune characteristics of this particular population, children infected with the novel coronavirus may present differences from adults regarding epidemiology, clinical symptoms, laboratory tests, and prognosis. There are few studies on children at present, with most identified through case reports (3). In order to make targeted clinical treatment strategies, more reliable studies are needed. Therefore, this study attempted to summarize and analyzed the clinical data of 11 children in the northeastern part of Chongqing who were admitted to Chongqing University Three Gorges Hospital, a designated hospital for the treatment of novel coronavirus infection in Chongqing. Through this, it is hoped that the reasons for the differences between pediatric patients and adult patients can be understood and effective prevention measures for children can be established. We present the following article in accordance with the STROBE reporting checklist (available at http://dx.doi.org/10.21037/tp-20-429).

\section{Methods}

\section{Participants}

From January 25 to February 29, 2020, the cases of 11 children who tested positive for COVID-19 and treated in Chongqing University Three Gorges Hospital were retrospectively analyzed and summarized. All children tested positive via viral nucleic acid tests of nasopharyngeal swabs, and the diagnosis of novel coronavirus infection was confirmed by two experts at the municipal and district levels. This study was approved by the ethics committee of Chongqing University Three Gorges Hospital. All procedures performed in this study involving human participants were in accordance with the Declaration of Helsinki (as revised in 2013). Individual consent for this retrospective analysis was waived.

\section{Contents}

\section{Clinical data collection}

The personal information, epidemiological history, clinical symptoms, chest computed tomography (CT) results, laboratory tests, treatment plan, and discharge situation of the pediatric patients were collected. Forms were made after approval by the two researchers. Laboratory tests included the first peripheral venous blood tests, high-sensitivity C-reactive protein (CRP), procalcitonin (PCT), lymphocyte subpopulation analysis, and inflammatory factors within 3 days after admission. Chest CT examinations were jointly reviewed by two professional radiologists with different titles, and the features, shapes, distribution, and density of lung lesions were analyzed and judged. All children's treatment plans were devised and implemented by experts at the municipal and district levels, and discharge standards were strictly followed in accordance with treatment guidelines. No cases lost to follow-up in the study.

\section{Diagnosis and classification}

The cases of the 11 infected children were clinically classified into asymptomatic, mild, common, severe, and critical in strict accordance with the "Novel Coronavirus Pneumonia Diagnosis and Treatment Protocol (Trial Versions 6)" (4), "Diagnosis and Prevention Suggestions for 2019 Novel Coronavirus Infection in Children (First Edition)" (5) and the "Pediatric Novel Coronavirus (SARS-Cov-2) Infection Diagnosis and Treatment Plan" (trial version from the Children's Hospital Affiliated with Chongqing Medical University).

\section{Statistical analysis}

Measurement data and enumeration data were presented as median and $\mathrm{n}(\%)$, respectively. Statistical analyses were 
done using the SPSS software (version 20.0).

\section{Results}

\section{Demographic data}

All 11 children with COVID-19 infection were from Northeast Chongqing. There were 7 males and 4 females, aged 7 months to 16 years, with a median age of 11 years and 5 months. There was 1 infant ( $<1$ year old), 1 toddler (1-4 years), 5 school-aged children (5-11 years), and 4 adolescents (12-18 years). Except for 1 patient who had a previous history of intestinal atresia surgery, poor digestive function, and frequent diarrhea, the remaining children were previously healthy and had no underlying disease. They were immunized according to standard scheduling.

\section{Epidemiological history}

Of the 11 children, 2 had lived in Wuhan for a long time and returned to Chongqing for the Spring Festival before the Wuhan lockdown. These 2 were considered imported cases. The remaining 9 cases involved familial cluster-onset with confirmed infections in the families, as shown in Table 1 .

\section{Clinical manifestations}

\section{Diagnosis and classification}

Among the 11 cases, 5 were asymptomatic (45\%), 2 were mild (18\%), 4 were normal (37\%), 0 were severe, and 0 were critical. Among them, the patient with underlying disease was normal.

\section{Clinical symptoms}

Of the 11 children, 5 had fever (45\%), with a course lasting 0.5 to 7 days, 4 patients had a fever before admission and a normal body temperature after admission, and 1 patient had a sustained low-moderate fever for 5 days after admission. There were 3 cases of slight dry cough (27\%), 1 case of sore throat (9\%), and 1 case of diarrhea (9\%). Among them, the patient with underlying disease presented with fever $(0.5$ days), cough, and diarrhea.

\section{Clinical signs}

Of the 11 patients, 7 did not show any positive signs of illness, but 4 patients had pharyngeal congestion on examination and thick breathing sounds in both lungs, but no wet or dry rales. The patient with underlying disease showed the above positive signs.

\section{Laboratory tests}

Routine blood examination showed normal white blood cell and lymphocyte counts in 11 cases, increased procalcitonin in 4 cases, and increased CRP in 1 case. Lymphocyte subgroup examination showed increased lymphocyte counts in 2 cases and decreased counts in 1 case; the percentages of $\mathrm{T}$ cells $(\mathrm{T} \%)$, cluster of differentiation 8 (CD8)+ $\mathrm{T}$ cells $(\mathrm{CD} 8+\mathrm{T} \%)$, and natural killer $(\mathrm{NK})$ cells $(\mathrm{NK} \%)$ were normal in 11 cases; the CD4+ T\% was increased in 2 cases, and the $\mathrm{CD} 4+\mathrm{T} \% / \mathrm{CD} 8+\mathrm{T} \%$ was decreased in 1 case. The percentage of $\mathrm{B}$ cells (B\%) was increased in 1 case. Regarding the detection of inflammatory factors, interleukin (IL-4), IL-10, and IL-17 were normal in 11 cases, while IL-6 was increased in 7 cases. Tumor necrosis factor alpha (TNF- $\alpha$ ) was increased in 1 case, and interferon gamma (IFN- $\gamma$ ) was increased in 6 cases, as shown in Table 2.

\section{Chest CT}

Of the 11 patients, 6 presented abnormal chest CT results, 2 of whom were asymptomatic. Three other clinically asymptomatic patients, however, had normal chest CT results. Regarding the lesion site, 1 case involved both lungs, 2 cases involved the lower lungs, 1 case involved the right upper lobe, and 2 cases involved the left lower lobe. Regarding lesion manifestations, 2 patients presented thickened textures, and 4 patients presented patchy ground-glass opacities which included the one with underlying disease. Regarding accompanying signs, no lymphadenopathy or pleural effusion was observed, as shown in Table 1.

\section{Treatment and efficacy}

All pediatric patients received antiviral supportive symptomatic treatment: 2 were treated with INF- $\alpha$ atomization alone (bid 12-14 days), 6 were treated with $\alpha$-interferon atomization combined with intravenous ribavirin (3-9 days), 3 were treated with $\alpha$-interferon atomization combined with lopinavir/ritonavir administered orally (9-13 days), and 1 was treated with azithromycin administered orally (5 days); no hormone therapy or tracheal intubation was administered. The patient with underlying disease was treated with $\alpha$-interferon atomization combined with intravenous 
Table 1 Clinical information of 11 children with COVID-19 infection

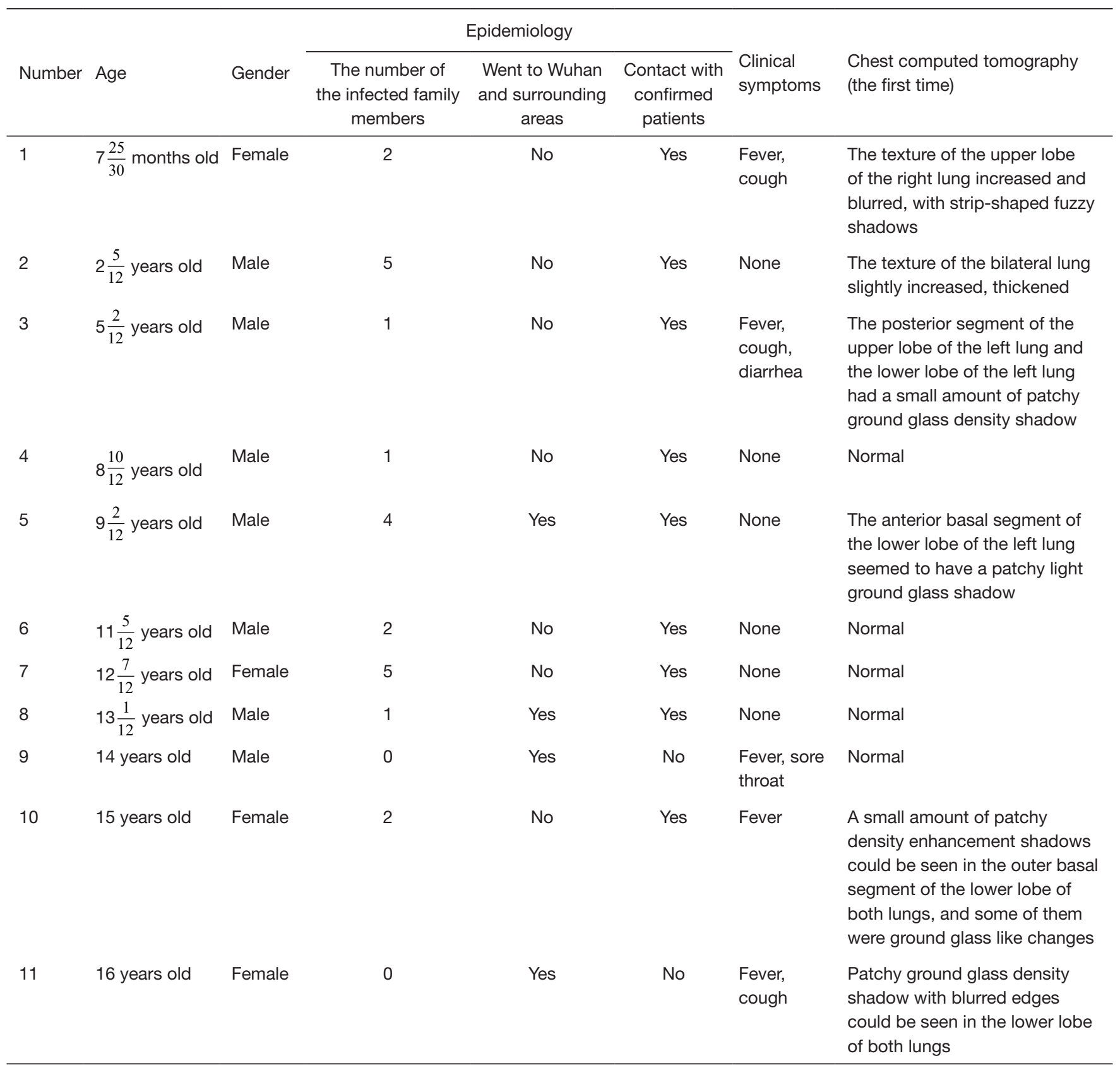

ribavirin; azithromycin was administered orally on the 11th day of hospitalization due to exacerbation of cough. All patients had been discharged from the hospital. The children's temperatures were normal, clinical manifestations were gone, lung lesions were absorbed and viral nucleic acid tests were negative for 2 consecutive times (interval of more than 24 hours). The length of stay ranged from 9 to 25 days. The patient with the underlying disease spent 25 days in hospital. So far, all patients have had negative re-examination results of viral nucleic acid tests.

\section{Discussion}

Coronaviruses are not novel phenomena in human society. A coronavirus is understood to be a single-stranded 


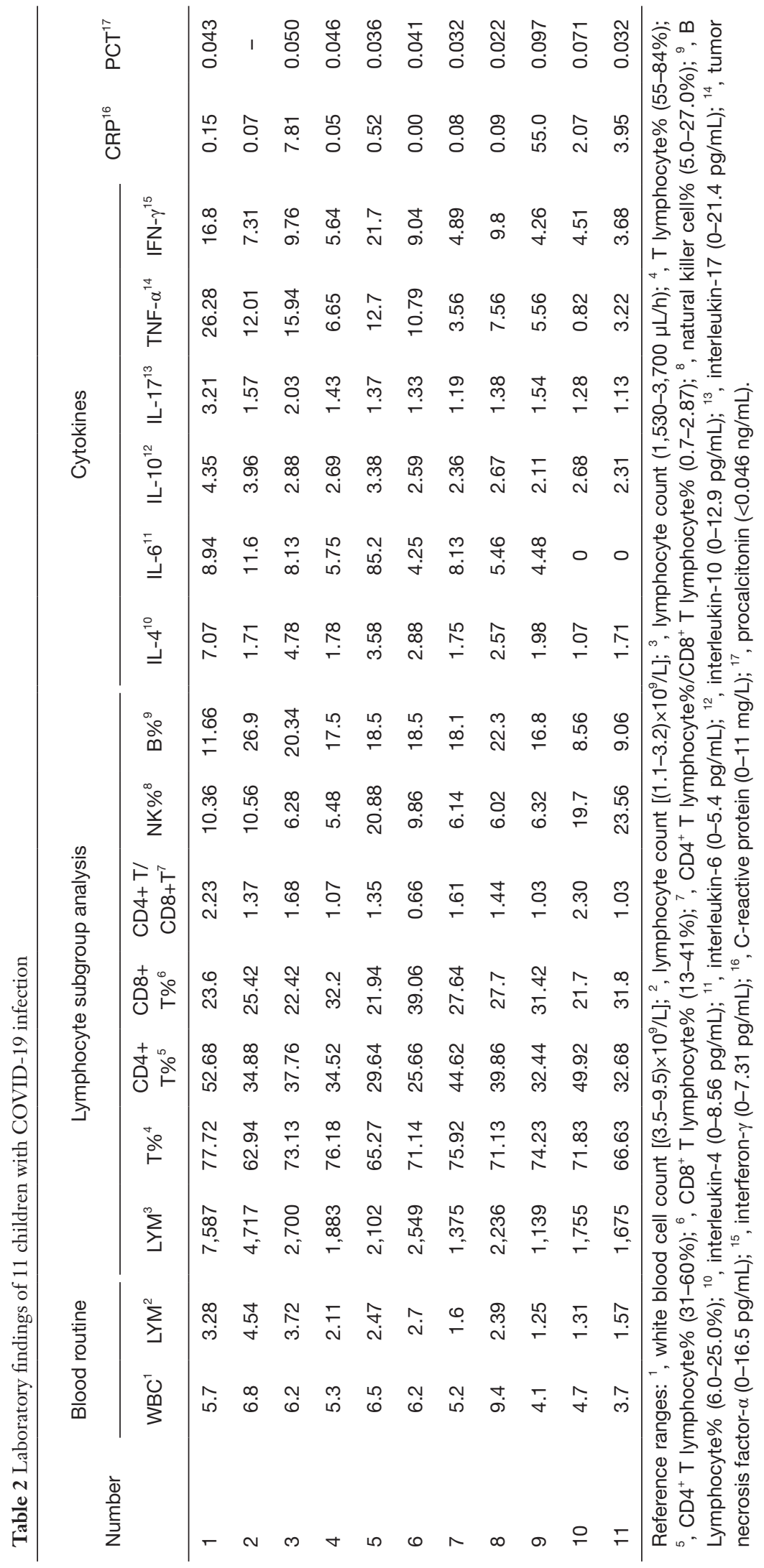


positive-strand RNA virus with an envelope structure that can infect mammals, including humans. It has highfrequency recombination ability and a high mutation rate; therefore, it easily adapts to new hosts. Prior to the discovery of COVID-19, there were 6 coronaviruses known to infect humans and cause respiratory diseases, 4 of which cause common upper respiratory tract infections, with the other 2 being severe acute respiratory syndrome coronaviruses [severe acute respiratory syndrome (SARS$\mathrm{CoV}), 2003$, and Middle East respiratory syndrome coronavirus (MERS-CoV), 2012], both of which are lethal (6). The new coronavirus has now spread globally, and the epidemic situation is severe. There is currently no specific drug for treating the virus.

Although all populations are generally susceptible to COVID-19 at present, the proportion of cases involving children is low according to official statistics, with most data coming from collections and case reports. As of February 21, 2020, there were a total of 76,718 confirmed COVID-19 cases worldwide, 7,264 suspected cases, and 2,247 deaths. But according to incomplete statistics, only about 300 confirmed cases involving children have been reported, with the children ranging in age from 30 hours to 17 years old (7). As a designated hospital for the treatment of novel coronavirus pneumonia in Chongqing, our hospital is responsible for the entire Northeast Chongqing area. From January 23 to 12:00 am on February 14, 2020, there were a total of 245 confirmed cases in this area, accounting for $45.5 \%$ of all confirmed cases in Chongqing. As of 12:00 am on February 29, 2020, our hospital had treated a total of 248 patients, of whom 39 had severe disease and 209 had minor disease, including 11 children, accounting for $4.4 \%$ of all confirmed cases. Among the 11 children with confirmed cases, 6 (55\%) were asymptomatic but had positive pharyngeal swab nucleic acid test results, and 1 (1/6) had an abnormal pulmonary CT result. These data are similar to those reported in a study published in Nature Medicine by the Guangzhou Women's and Children's Medical Center on March 13, which reported that based on nucleic acid tests performed on 745 children and 3,174 adults with a history of close contact or with confirmed family members with COVID-19, only 10 children were positive $(1.3 \%$ of the total children), compared with 111 adults $(3.5 \%)$ with positive results, which is a 2.7 -fold difference (8). Compared with adults, children who carry the novel coronavirus are not only small in number but also exhibit mild clinical manifestations, with unobvious and atypical symptoms.

The definition of "child" implies a wide range of ages, from 28 days to 18 years. Based on growth and development characteristics, children can be divided into several stages. Because younger infants and toddlers have a limited range of motion, they have the most contact with their families in their daily activities. In this group of patients, except for two adolescents who were studying and living in Wuhan, the others were in close contact with family members or relatives who were carrying the virus, and it was discovered that the disease onset in children was not necessarily later than that in adults. Among these patients, the youngest became ill after coming in contact with an aunt who returned from Wuhan; the aunt had been infected with the virus and was asymptomatic. Her infection was revealed only after routine screening for the disease because of the child patient. Two children had a clear history of living in the epidemic area. When novel coronavirus infection involving young children is suspected at an outpatient clinic, it is necessary to note family clustering characteristics and routinely screen out those who were in close contact with the children and identify potential asymptomatic carriers.

The most common manifestations of novel coronavirus infection in children are fever and cough, and other symptoms, such as fatigue, diarrhea, myalgia, nasal congestion, and runny nose are not common $(9,10)$. There are no other particular symptoms. One patient in this group had diarrhea but had a previous history of gastrointestinal deformity surgery and had habitually suffered from diarrhea previous to diagnosis. Therefore, diarrhea is not a specific symptom of novel coronavirus infection. Among these pediatric cases, the duration of fever was short. Most of the patients had a normal body temperature after admission and showed only a brief fever before admission. The maximum fever duration was 7 days, which did not exceed the maximum 8 days described in the guidelines $(5,11)$. Relevant symptoms returned to normal soon after symptomatic treatment was provided. Severe adult cases progress rapidly, and most involve dyspnea. Severe cases quickly develop into acute respiratory distress syndrome (ARDS), metabolic acidosis that is hard to correct, and coagulation dysfunction; in particular, the elderly with chronic diseases usually constitute the more severe cases (12). However, there have been no cases of severe or critically ill children among the severe cases in this center, supporting the description in the guidelines stating that infected children generally have mild symptoms. 
The patients with abnormal chest CT results in this group mainly showed patchy ground-glass opacities near the pleural area, and the lesions were more localized. The manifestations were not what is typically observed in adults, without a diffuse distribution, pleural effusion, or hilar lymphadenopathy $(13,14)$. Two patients had no clinical manifestations but showed abnormal chest CT results and positive viral nucleic acid tests (throat swab). One patient had clinical manifestations and a positive viral nucleic acid test (throat swab) but a normal chest CT. Therefore, there is no clear relationship between clinical manifestations of infection and the timing of chest imaging changes. The probability of false-negative nucleic acid tests currently indicates that clinical diagnosis needs to be complemented with chest imaging data (15). In addition, in this group of cases, the imaging of the lungs in two younger children was atypical, showing a coarse and fuzzy lung texture that was difficult to distinguish from other pathogenic pneumonia-related manifestations. A combination of epidemiology and nucleic acid detection is required for diagnosis.

At the early stage after novel coronavirus infection, routine venous blood tests in adults often indicate a decrease in lymphocyte counts (16). Approximately $35 \%$ to $82.1 \%$ of adult patients present a decrease in lymphocyte count, and $86 \%$ present an increase in CRP. Some studies have found abnormal levels of lymphocyte subsets in patients with COVID-19, which manifests as a decline in NK and CD4+ T cells. The decrease in the total number of lymphocytes in severe patients mainly manifests as a decrease in CD4+ $\mathrm{T}$ lymphocytes, accompanied by a decrease in the CD4+/CD8+ ratio $(17,18)$. In all children in this group, the peripheral blood lymphocyte count was normal in the first routine blood test, and the proportion of NK cells was normal in the subgroup analysis. CD4+ $\mathrm{T}$ cells were increased in 2 patients, and the CD4+/ CD8+ ratio was decreased in only 1 . In critically ill adult patients, the immune system is disrupted, and the number of lymphocytes continues to decrease; a large number of inflammatory factors, such as IL-6, IL-10, IL-12, and IFN- $\gamma$, will be generated inside the body, and a cytokine storm will occur. This is a severe systemic reaction triggered by the excessive activation of the immune system caused by infection, which can result in multiple organ failure in patients or even death. This is one reason severe illness rapidly develops into critical illness (19). In our cases, IL-4, IL-10, and IL-17 were normal in 11 children, while IL-6 was increased in 7 patients (64\%), TNF- $\alpha$ was increased in 1 patient (9\%), and IFN- $\gamma$ was increased in 6 patients $(55 \%)$. Although there were also elevated inflammatory factors, they did not cause a cytokine storm. The human immune network system is very complex. In the process of the identification and elimination of pathogens, various immune cells and molecules coordinate and mutually react, ultimately maintaining the stability of the body's immune system and eliminating pathogens. When a novel coronavirus invades, to initiate virus replication, it binds to the angiotensin-converting enzyme 2 (ACE2) receptor on the surface of human alveolar epithelial cell membranes, resulting in damage to the body. Then, macrophages, especially dendritic cells, recognize and engulf the virus and destroy it. Additionally, the virus information is transmitted to $\mathrm{T}$ cells, and various antiviral factors, chemokines and cytotoxic molecules are produced, stimulating the proliferation and differentiation of the cells involved in the immune response, improving their functions, and inhibiting virus replication and clearing the virus. These factors also directly or indirectly damage human cells and tissues, resulting in various clinical symptoms, such as fever, cough, diarrhea, among others. B lymphocytes can regulate $T$ cell subsets and the functions of dendritic cells and can also activate $\mathrm{T}$ cells. Therefore, the occurrence, development, and outcome of the disease after the human body is infected with novel coronavirus depends on the interaction between the body's immune cells and the virus. At present, the population is generally susceptible. From analyzing the transmission route of COVID-19, young children and adults almost have the same rate of infection through close contact (20), but the overall number of cases is significantly different, which may be because children are more likely to undergo immunological adaptation. This is an optimal immune response: for one, immune cells can clear pathogens; for another, there is no severe inflammatory response, and the child becomes an asymptomatic carrier or has mild clinical symptoms. This is different from the high incidence of infection in adults. In particular, in elderly men with multiple underlying diseases, the host immune cells are extremely stressed and are more prone to severe inflammatory reactions, more severe symptoms, and higher mortality (21).

In addition to the differences in social aggregation between children and adults, the lower incidence, milder symptoms, and shorter durations of the disease are still more prominent in children, which does not rule out the existence of a physiological basis of insusceptibility. The prevalence of SARS in children is also lower than 
that in adults. Considering the common characteristics of coronaviruses, children may have a cellular structure or immunity basis which can account for the relative insusceptibility (22). To date, the epidemic is still spreading. Although the control of the epidemic in China has seen significant progress, the epidemic situation in the world is generally still severe. There are currently no specific antiviral drugs, and vaccines are still being developed. Even if there have been no reported fatalities in children with COVID-19 to date, the possible risk of death cannot be ignored. It is still necessary to follow the current national prevention and control measures: control the source of infection, cut off the route of transmission, protect vulnerable groups, and protect children. In addition, we can learn from the characteristics of children's immune systems and investigate their unusual features, thereby facilitating the prevention and treatment of the disease.

This is a single-center retrospective study. The sample size is small but representative, and the data are true and reliable; however, the research results have a certain degree of bias. Considering the small number of children diagnosed at present (23), this study may still serve as an important reference.

\section{Acknowledgments}

Our deepest gratitude goes to the anonymous reviewers and editors for their careful work and thoughtful suggestions that have helped improve this paper substantially.

Funding: None.

\section{Footnote}

Reporting Checklist: The authors have completed the STROBE reporting checklist. Available at http://dx.doi. org/10.21037/tp-20-429

Data Sharing Statement: Available at http://dx.doi. org/10.21037/tp-20-429

Conflicts of Interest: All authors have completed the ICMJE uniform disclosure form (available at http://dx.doi. org/10.21037/tp-20-429). The authors have no conflicts of interest to declare.

Ethical Statement: The authors are accountable for all aspects of the work in ensuring that questions related to the accuracy or integrity of any part of the work are appropriately investigated and resolved. This study was approved by the ethics committee of Chongqing University Three Gorges Hospital. All procedures performed in this study involving human participants were in accordance with the Declaration of Helsinki (as revised in 2013). Individual consent for this retrospective analysis was waived.

Open Access Statement: This is an Open Access article distributed in accordance with the Creative Commons Attribution-NonCommercial-NoDerivs 4.0 International License (CC BY-NC-ND 4.0), which permits the noncommercial replication and distribution of the article with the strict proviso that no changes or edits are made and the original work is properly cited (including links to both the formal publication through the relevant DOI and the license). See: https://creativecommons.org/licenses/by-nc-nd/4.0/.

\section{References}

1. Zhu N, Zhang D, Wang W, et al. A Novel Coronavirus from Patients with Pneumonia in China, 2019. N Engl J Med 2020;382:727-33.

2. Jiang RM. Process of guidelines for diagnosis and treatment of corona virus disease 2019 trail version 1-6. Chin J Infect Dis 2020;38:129-33.

3. Long XR, Zhu J, Zhao RQ, et al. Epidemiology and clinical features of highly pathogenic human coronavirus infection in children. Zhonghua Er Ke Za Zhi 2020;58:351-4.

4. National Health Commission, National Administration of Traditional Chinese Medicine. Guidelines on diagnosis and treatment of novel coronavirus pneumonia (Trial sixth edition). Chin J Infect Control Vol 2020;19:192-5.

5. The Society of Pediatrics, Chinese Medical Association; the Editorial Board, Chinese Journal of Pediatrics. Recommendations for the diagnosis, prevention and control of the 2019 novel coronavirus infection in children (first interim edition). Chin J Pediatr 2020;58:169-74.

6. Paules CI, Marston HD, Fauci AS. Coronavirus Infections-More Than Just the Common Cold. JAMA 2020;323:707-8.

7. Ma J, Shen LL, Liu HM, et al. Interpretation of prevention and control recommendations during the period for preventing and controlling COVID-19 in children. Chongqing Medicine 2020;49:2816-9.

8. Xu Y, Li XF, Zhu B, et al. Characteristics of pediatric 
SARS-CoV-2 infection and potential evidence for persistent fecal viral shedding. Nat Med 2020;26:502-5.

9. Liu W, Zhang Q, Chen J, et al. Detection of Covid-19 in Children in Early January 2020 in Wuhan, China. N Engl J Med 2020,382:1370-1.

10. Chan JF, Yuan S, Kok KH, et al. A familial cluster of pneumonia associated with the 2019 novel coronavirus indicating person-to-person transmission: a study of a family cluster. Lancet 2020;395:514-23.

11. Wang D, Ju XL, Xie F, et al. Clinical analysis of 31 cases of 2019 novel coronavirus infection in children from six provinces (autonomous region) of northern China. Zhonghua Er Ke Za Zhi 2020;58:269-74.

12. Huang C, Wang Y, Li X, et al. Clinical features of patients infected with 2019 novel coronavirus in Wuhan, China. Lancet 2020;395:497-506.

13. Zhou Y, Yang GD, Feng K, et al. Clinical features and chest CT findings of coronavirus disease 2019 in infants and young children. Zhongguo Dang Dai Er Ke Za Zhi 2020;22:215-20.

14. Chung M, Bernheim A, Mei X, et al. CT Imaging Features of 2019 Novel Coronavirus (2019-nCoV). Radiology 2020;295:202-7.

15. Li Z, Yi Y, Luo X, et al. Development and Clinical Application of A Rapid IgM-IgG Combined Antibody Test for SARS-CoV-2 Infection Diagnosis. J Med Virol 2020;92:1518-24.

16. Wang D, Hu B, Hu C, et al. Clinical Characteristics of 138 Hospitalized Patients With 2019 Novel Coronavirus-

Cite this article as: Chen Q, Tian X, Luo Y, Liu J, Jiang L, Feng X, Chen Z. Epidemiological and clinical characteristics analysis of 11 children with 2019 novel coronavirus infection in Chongqing: a single-center retrospective study. Transl Pediatr 2020;9(6):818-826. doi: 10.21037/tp-20-429
Infected Pneumonia in Wuhan, China. JAMA 2020;323:1061-9.

17. Li Q, Nie K, Qiao ZR, et al. Expression of lymphocyte subsets in peripheral blood of patients with novel coronavirus pneumonia and its clinical significance. Int J Lab Med 2020;41:2092-5.

18. Lu ZL, He RY, Jiang WY, et al. Clinical characteristics and immune function analysis of COVID-19. Medical Journal of Wuhan University 2020;41:529-32, 546.

19. Wang XY, Liao J, Chen J. Research progress on immunological features and immunotherapy strategies of coronavirus disease 2019. Journal of Tropical Medicine 2020;20:571-5, 602 .

20. Bi Q, Wu Y, Mei S, et al. Epidemiology and Transmission of COVID-19 in 391 cases and 1,286 of their close contacts in Shenzhen, China: a retrospective cohort study. Lancet Infect Dis 2020;20:911-9.

21. Yang XQ, Zhao XD. Associativity from Children with Corona Virus Disease 2019. Journal of Pediatric Pharmacy 2020;26:1-4.

22. Yang BB, Zhu YL. Epidemiology and treatment of novel coronavirus pneumonia in children and adults. Journal of Xi'an Jiaotong University (Medical Sciences) 2020;41:483-7.

23. Wang JG, Cui HR, Tang HB, et al. Gastrointestinal symptoms and fecal nucleic acid testing of children with 2019 coronavirus disease: a systematic review and metaanalysis. Sci Rep 2020;10:17846.

(English Language Editor: J. Gray) 